Www.jmscr.igmpublication.org

Impact Factor (SJIF): 6.379

Index Copernicus Value: 79.54

ISSN (e)-2347-176x ISSN (p) 2455-0450

crossrefDOI: https://dx.doi.org/10.18535/jmscr/v6i10.82

Journal Of Medical Science And Clinical Research

\title{
A Cross sectional Study on role of MRI and its Clinico-Radiological correlation of Patients with White Matter Disease
}

Authors
Dr Saif Rahman ${ }^{1}$, Dr Ahmad Rizwan Karim ${ }^{2}$, Dr Annugya Mimansa
${ }^{1,3}$ Post Graduate Trainee, Department of Radiodiagnosis, Katihar Medical College and Hospital, Katihar, Bihar, India
${ }^{2}$ Professor, Department of Radiodiagnosis, Katihar Medical College and Hospital, Katihar, Bihar, India
Corresponding Author
Dr Saif Rahman
Post Graduate Trainee, Department of Radiodiagnosis, Katihar Medical College and Hospital, Katihar, Bihar, India
Email: drsaifrahman123@gmail.com, Contact: +91 9711069834
Abstract
Objectives: our study was to evaluate the clinico and radiological correlation and to find the role of MRI
as an investigative modality for diagnosis of patients with white matter diseases.
Methodology: A total of 30 cases of white matter lesions were selected for this study. A detail history,
clinical examination and relevant investigations were performed to all cases. All the patients were examined
with 1.5 Tesla systems (Toshiba Medical System). Head coil was used in all the patients. A combination of
T1 and T2 weighted and FLAIR sequences and post gadolinium T1 weighted was obtained in each patient.
Results: Data was analyzed by using simple statistical methods with the help of MS-Office software.
Conclusions: Multiple Sclerosis was the most common white matter disease. Female was more
preponderance than male. Patients with second or third decade of life were commonly suffered with MS.
Acute Disseminated Encephalomyelitis and Progressive Multifocal Leukoencephalopathy were the second
most common disorders of white matter. Cerebral white matters with asymmetric and patchy involvement
were seen in all cases of ADEM. Hence, MRI is one of the best invaluable choices for the detection of white
matter diseases.
Keywords: White matter disease, Multiple sclerosis, ADEM, MRI.

\section{Introduction}

Multifocal white matter abnormalities are characteristic of multiple sclerosis (MS), a primary demyelinating disease. Multiple sclerosis most commonly affects females, and is most prevalent in the 20-40 year old age group. In middle aged adults, the female-to-male radio is $1.5-2: 1$, but in younger patients the female preponderance is greater. ${ }^{[1]}$ Although $75 \%$ of patients with multiple sclerosis present between the ages of 20 and 50 years of age, it is important to bear in mind that $15 \%$ present in the first and second decade, and $10 \%$ present after 50 years of age. A negative brain MR does not exclude multiple sclerosis, as the disease may present with spinal cord involvement only (with a normal cranial MR). In patients with intracranial involvement, the periventricular white matter is most commonly involved, usually with an asymmetrical distribution. ${ }^{[1]}$

Magnetic resonance imaging (MRI) was found to be more sensitive than computed tomography 
(CT) in the detection of (white matter) lesions of the brain. Following initial enthusiasm about the new diagnostic possibilities, like in multiple sclerosis (MS), ${ }^{[2]}$ it was reported that clinically silent (incidental) magnetic resonance (MR) lesions could also be found in normal ageing and a variety of other diseases. ${ }^{[3]}$ During a period of scepticism about the apparent lack of specificity of MR in the diagnosis of brain lesions, the term UBO (unidentified bright object) was employed to describe incidentally discovered brain abnormalities. In the second half of the 1980s, further studies on white matter lesions (WML) in a variety of diseases increased our knowledge about the prevalence of incidental MR lesions and risk factors for such lesions. Improved image quality, newer MR techniques (including gadolinium enhancement), and knowledge about the shape, size and distribution of incidental MR lesions have provided further clues to the differential diagnosis of (incidental) MR lesions. ${ }^{[4]}$ Aim of our study was to evaluate the role of MRI as an investigative modality for diagnosis of white matter diseases and its value in early diagnosis and management and its clinico-radiological correlation of patients with white matter disease.

\section{Materials \& Methods}

This present study was conducted in department of Radiodiagnosis, Kathihar Medical College and Hospital, Katihar, Bihar, India during a period from January 2017 to February 2018. Attendants of entire subject signed an informed consent approved by institutional ethical committee of Katihar Medical College and Hospital, Katihar was sought.
Selection of data: A total of 30 cases of white matter disease with irrespective of sex were enrolled in this study. Age group of cases were 7 to 75 years. Male and female ratio was 17:13.

\section{Methods}

A detail history, clinical examination and relevant investigation were performed to all cases.

All the patients were examined with 1.5 Tesla systems (Toshiba Medical System). Head coil was used in all the patients. A combination of $\mathrm{T} 1$ and T2 weighted and FLAIR sequences and post gadolinium T1 weighted was obtained in each patient. Each MR image was interpreted by two Radiologists proficient in reporting brain MRI. In case of difference of opinion, consensus was developed through discussion between the two radiologists. The diagnosis was confirmed by laboratory findings of CSF oligoclonal band in patients with MS. Among cases of acute disseminated encephalomyelitis and leukodystrophies, the diagnosis was established through typical imaging findings and clinical course without any relapse.

\section{Statistical Analysis}

Data was analyzed by using simple statistical methods with the help of MS-Office software.

\section{Results}

In this present study, we were included a total 30 cases of white matter disease. Patient with age group was $7-75$ years. Out of 30 cases, $17(56.67 \%)$ cases were males and $13(43.33 \%)$ cases were females.

Table.1. Distribution of white matter disease $(\mathrm{N}=30)$

\begin{tabular}{|c|c|c|c|c|}
\hline Diseases & \multicolumn{2}{|c|}{ Sex } & No. of cases & $\%$ \\
\hline \multirow[t]{2}{*}{ Multiple sclerosis } & $\mathrm{M}$ & $\mathrm{F}$ & \multirow[b]{2}{*}{10} & \multirow[t]{2}{*}{$33.33 \%$} \\
\hline & $4(40 \%)$ & $6(60 \%)$ & & \\
\hline \multirow[t]{2}{*}{ Acute disseminated encephalomyelitis } & $\mathrm{M}$ & $\mathrm{F}$ & & \multirow[t]{2}{*}{$20 \%$} \\
\hline & $4(66.67 \%)$ & $2(33.33 \%)$ & 6 & \\
\hline \multirow[t]{2}{*}{ Progressive multifocal leukoencephalopathy } & $\mathrm{M}$ & $\mathrm{F}$ & & \multirow[t]{2}{*}{$10 \%$} \\
\hline & $2(66.67 \%)$ & $1(33.33 \%)$ & 3 & \\
\hline \multirow[t]{2}{*}{ Central pontine myelinolysis } & $\mathrm{M}$ & $\mathrm{F}$ & & \multirow[t]{2}{*}{$6.66 \%$} \\
\hline & $1(50 \%)$ & $1(50 \%)$ & 2 & \\
\hline Radiation injury & $1(100 \%)$ & 0 & 1 & $3.33 \%$ \\
\hline
\end{tabular}




\begin{tabular}{|l|c|c|c|c|}
\hline \multirow{2}{*}{ Leukodystrophies } & $\mathrm{M}$ & $\mathrm{F}$ & \multirow{2}{*}{2} & $6.66 \%$ \\
\cline { 2 - 3 } & $1(50 \%)$ & $1(50 \%)$ & 2 & \\
\hline Periventricular leukomalacia & 0 & $1(100 \%)$ & & $3.33 \%$ \\
\hline \multirow{2}{*}{ Diffuse axonal injury } & $\mathrm{M}$ & $\mathrm{F}$ & \multirow{2}{*}{2} & $6.66 \%$ \\
\cline { 2 - 3 } & $1(50 \%)$ & $1(50 \%)$ & 2 & $3.33 \%$ \\
\hline Reversible posterior leukoencephalopathy & $1(100 \%)$ & 0 & 1 & $6.66 \%$ \\
\cline { 2 - 3 } Motor neuron disease & $\mathrm{M}$ & $\mathrm{F}$ & 2 & \\
\cline { 2 - 3 } & $2(100 \%)$ & 0 & 30 & $100 \%$ \\
\hline Total & $17(56.67 \%)$ & $13(43.33 \%)$ & 2 \\
\hline
\end{tabular}

In this study, multiple sclerosis was the most common white matter disease. Out of 30 cases of white matter disease, $10(33.33 \%)$ cases was the multiple sclerosis. Male 4(40\%) and females $6(60 \%)$ were suffered with multiple sclerosis. Male and female ratio was 2:3.

Mean age was 31.2 years. Average age of female cases was 25 years and male cases were 36 years of patients with multiple sclerosis.

In this present study, out of 30 cases of white matter disease, $6(20 \%)$ cases were encountered of acute disseminated encephalomyelitis. 4(66.67\%) cases were male and $3(33.33 \%)$ cases were female.

$3(10 \%)$ cases were of progressive multifocal leukoencephalopathy. All the three patients presented with motor symptoms in the form of hemiparesis and one patient had cerebellar signs in addition. On MR, one patient showed patchy hypointense lesions on T1WI in subcortical white matter of right occipital and parietal lobe, which were hyperintense on T2WI and FLAIR sequences.

Central pontine myelinolysis and leukodystrophies cases were 2(6.66\%). Male and female ratio was $1: 1$. One of them had definite predisposing factors like hyponatraemia and the other patient was alcoholic. Both the patients showed extra pontine involvement in addition to the characteristic involvement of central pons.

$1(3.33 \%)$ case of Periventricular Leucomalacia (PVL): Patient was an 8-year-old female child presented with history of preterm delivery and both asphyxia with microcephaly. At present, the patient has presented with mental retardation and seizures. MRI showed loss of white matter volume and bilateral symmetrical hyperintensity of the periventricular white matter especially of the periatrial region. $1(3.33 \%)$ male case was of radiation injury. $2(6.66 \%)$ male cases were encountered with motor neuron disease.

Table.2. Presenting complains of MS ( $=10)$.

\begin{tabular}{|l|c|c|}
\hline Symptoms & No. of cases & Percentage \\
\hline Visual loss & 8 & $80 \%$ \\
\hline Weakness & 5 & $50 \%$ \\
\hline Sensory loss & 2 & $20 \%$ \\
\hline Ataxia & 2 & $20 \%$ \\
\hline Seizures & 1 & $10 \%$ \\
\hline Facial palsy & 2 & $20 \%$ \\
\hline
\end{tabular}

In this present study, most common symptom of multiple sclerosis was visual impairment $8(80 \%)$ followed by $5(50 \%)$ weakness, $2(20 \%)$ sensory loss, ataxia and facial palsy and $1(10 \%)$ of seizures.

Table.3. Symptoms of acute disseminated encephalomyelitis $(\mathrm{N}=6)$.

\begin{tabular}{|l|c|c|}
\hline Symptoms & $\begin{array}{c}\text { No. of } \\
\text { cases }\end{array}$ & Percentage \\
\hline Altered consciousness & 4 & $66.67 \%$ \\
\hline Motor symptoms & 2 & $33.33 \%$ \\
\hline Urinary retention & 2 & $33.33 \%$ \\
\hline MRI appearance & 6 & $100 \%$ \\
\hline $\begin{array}{l}\text { Cerebral white matter with } \\
\text { asymmetric and patchy } \\
\text { involvement }\end{array}$ & 2 & $33.33 \%$ \\
\hline Brain stem involvement & 1 & $16.67 \%$ \\
\hline $\begin{array}{l}\text { Spinal cord and cerebellar } \\
\text { Involvement }\end{array}$ & 1 & $16.67 \%$ \\
\hline Thalamic involvement & & \\
\hline
\end{tabular}

All cases 6(20\%) of Acute Disseminated Encephalomyelitis (ADEM) were a history of fever prior to the onset of clinical symptoms. Ages were varied to 6-75 years. Out of total 6 cases of ADEM, most common symptoms were $4(66.67 \%)$ altered consciousness followed by $2(33.33 \%)$ motor symptoms and urinary retention. 
On MRI appearance, all cases of ADEM were shown cerebral white matter with asymmetric and patchy involvement. 2 (33.33\%) were shown brain stem involvement. And 1(16.67\%) case was shown spinal cord, cerebral involvement and thalamic involvement.

\section{Discussion}

MRI is an important noninvasive imaging modality which has a very high sensitivity for detecting white matter lesions due to its excellent gray-white matter resolution. Multiplanar imaging is possible only with MRI, which helps in the detection and localization of lesions. ${ }^{[5]}$

In this present study, we were enrolled a total of 30 cases with age group of 7 to 75 years of white matter disease. $17(56.67 \%)$ cases were male and $13(43.33 \%)$ cases were female. Multiple sclerosis was the most common disease of all the white matter disease. Male and female ratio was 2:3. Multiple sclerosis was more preponderance in female than male. Mean age was 31.2 years. In female average age was 25 years and in male average age was 36 years. Most common symptom of multiple sclerosis was visual impairment $8(80 \%)$ followed by $5(50 \%)$ weakness, 2(20\%) sensory loss, ataxia and facial palsy and $1(10 \%)$ of seizures. MR imaging findings include all patients showing bilateral periventricular hyperintensities on $\mathrm{T} 2$ weighted and FLAIR images. Deep grey matter hyperintensities were also identified in all patients. Two patients showed hyperintense lesions in bilateral frontal, parietal and occipital lobes, each lesion measuring approximately 5-10 $\mathrm{mm}$ in size.

Similar study was conducted by BN Lakhkar, et al. (2002) ${ }^{[6]}$ and stated that Demyelinating and dysmyelinating white matter diseases are important components of neurological problems. White matter diseases include Multiple Sclerosis (MS), Leukodystrophies, Central Pontine Myelinolysis, Acute Disseminated Encephalomyelitis and Progressive Multifocal Leukoencephalopathy. MS is the commonest of all the white matter diseases.
Dr. Sindu P. Gowdar, et al. (2015) ${ }^{[5]}$ also found that female was more preponderance than male with multiple sclerosis. ${ }^{[5]}$

In this present study, out of 30 cases of white matter disease, $6(20 \%)$ cases were encountered of acute disseminated encephalomyelitis. 4(66.67\%) cases were male and $3(33.33 \%)$ cases were female. Among cases of acute disseminated encephalomyelitis and leukodystrophies, the diagnosis was established through typical imaging findings and clinical course without any relapse. On MRI, Asymmetrical hyperintense signals in the subcortical white matter of frontal, parietal and occipital lobe and cerebellum with enhancement on post contrast scans were seen in the cases of ADEM.

$2(33.33 \%)$ cases were shown brain stem involvement. And 1(16.67\%) case was shown spinal cord, cerebral involvement and thalamic involvement

All cases 6(20\%) of Acute Disseminated Encephalomyelitis (ADEM) were a history of fever prior to the onset of clinical symptoms. Ages were varied to 6-75 years. Out of total 6 cases of ADEM, most common symptoms were $4(66.67 \%)$ altered consciousness followed by $2(33.33 \%)$ motor symptoms and urinary retention.

$3(10 \%)$ cases were of progressive multifocal leukoencephalopathy. . All the three patients presented with motor symptoms in the form of hemiparesis and one patient had cerebellar signs in addition. On MR, one patient showed patchy hypointense lesions on T1WI in subcortical white matter of right occipital and parietal lobe, which were hyperintense on T2WI and FLAIR sequences.

Central pontine myelinolysis and leukodystrophies cases were 2(6.66\%). Male and female ratio was $1: 1$. One of them had definite predisposing factors like hyponatraemia and the other patient was alcoholic. Both the patients showed extra pontine involvement in addition to the characteristic involvement of central pons.

Humera Ahsan, et al.(2008) ${ }^{[7]}$ were stated that leukodystrophies, which include Metachromatic 
Leukodystrophy and Adrenoleukodystrophy, Alexender's and Niemen Pick Diseases, were the other most common group of white matter diseases. In their results showed those $10(28.6 \%)$ patients, 7 (70\%) males and 3 (30\%) females, suffered form leukodystrophies. Each type of leukodystrophy presented with different frequencies and MRI findings.

In this present study, 1(3.33\%) case of Periventricular Leucomalacia (PVL): Patient was an 8-year-old female child presented with history of preterm delivery and both asphyxia with microcephaly. At present, the patient has presented with mental retardation and seizures. MRI showed loss of white matter volume and bilateral symmetrical hyperintensity of the periventricular white matter especially of the periatrial region. $1(3.33 \%)$ male case was of radiation injury. 2(6.66\%) male cases were encountered with motor neuron disease.

Ashikaga $\mathrm{R}$ et al (1996) ${ }^{[8]}$ found that FLAIR sequences had a better sensitivity for subtle demyelinating foci especially those with periventricular locations. The most common site of lesion in this study was the periventricular area. Fazekas, et al. (1993) ${ }^{[9]}$ and Jena AN, et al. (1991) ${ }^{[10]}$ have also noted the periventricular area as the commonest location.

BN Lakhkar, et al. (2002) $)^{[6]}$ conducted a study in which four cases of periventricular Leukomalacia (PVL) were reported. In their study, two had a history of preterm delivery $\&$ asphyxia while the remaining two were full term infants with insult in the prenatal life. The most common clinical presentation was spastic diplegia (cerebral palsy) followed by seizures. MRI showed loss of white matter volume and bilateral symmetrical hyperintensity of the periventricular white matter especially of the periatrial region in all patients. Ventriculomegaly and scalloping of ventricular margins were seen in two patients. They opined that the typical imaging findings include peritrigonalhy perintensities on T2WI, focal ventricular enlargement and irregular, scalloped ventricular contours. White matter volume is reduced and the posterior corpus callosum appears moderately atrophic.

\section{Conclusion}

Multiple Sclerosis was the most common white matter disease. Female was more preponderance than male. Patients with second or third decade of life were commonly affected with MS. Second most common disorders of white matter were acute disseminated encephalomyelitis and progressive multifocal leukoencephalopathy. Cerebral white matters with asymmetric and patchy involvement were seen in all cases of ADEM. Hence, MRI is one of the best invaluable choices for the diagnosis of white matter disease.

\section{References}

1. Blake A. Johnson. A Practical Approach to White Matter Disease. Advanced MRI 2002 - from head to toe.

2. Young IR, Hall AS, Pallis CA, Legg NJ, Bydder GM, Steiner RE: Nuclear magnetic resonance imaging of the brain in multiple sclerosis. Lancet. 1981;ii:1063-1066.

3. Zimmerman RD, Fleming CA, Lee BCP, Saint-Louis LA, Feck MD: Periventricular hyperintensity as seen by magnetic resonance: Prevalence and significance. AJNR 1986;7:13-20.

4. Frederik Barkhof, Philip Scheltens. Imaging of White Matter Lesions. Cerebrovasc Dis 2002;13(suppl 2):21-30.

5. Dr. Sindu P. Gowdar, Dr. PramodSetty J.,Dr. Naveen S. Maralihalli, Dr. Jeevika.M.U., Dr. Mohd Kamran Siddiqui. IOSR Journal of Dental and Medical Sciences (IOSR-JDMS). Apr. 2015; 14(4): 26-44.

6. BN Lakhkar, M Aggarwal, JR John. MRI in White Matter Diseases - Clinico Radiological Correlation. Ind J Radiol Imag 2002 12:1:43-50.

7. Humera Ahsan, Muhammad Zafar Rafique, Fozia Ajmal, Mohsin Wahid, Muhammad Azeemuddin, Farah Iqbal. 
Magnetic Resonance Imaging (MRI) findings in White Matter Disease of Brain. J Pak Med Assoc. March 2008; 58: 2.

8. Francis GS, Evans AC, Arnold DL. Neuroimaging in multiple sclerosis. Neurologic Clinics 1995;13(1):147-171.

9. Ashikaga $R$, Araki $Y$, Ishida O. MR FLAIR imaging of herpes simplex encephalitis. Radiat Med 1996;14(6):349352.

10. Offenbacher H, Fazekas F, Schmidt R, et al. Assessment of MRI criteria for a diagnosis of MS. Neurology 1993;43(5):905-909. 